\title{
Familial monophasic acute transverse myelitis due to the pathogenic variant in VPS37A
}

Maureen A. Mealy, RN, Tai-Seung Nam, MD, PhD, Santiago J. Pardo, BA, Carlos A. Pardo, MD, PhD, Nara L. Sobreira, MD, PhD, Dimitrios Avramopoulos, MD, PhD, David Valle, MD, Kathleen H. Burns, MD, PhD, and Michael Levy, MD, PhD

Neurol Genet 2018;4:e213. doi:10.1212/NXG.0000000000000213

\section{Abstract}

\section{Objective}

To identify genetic differences among siblings with a family history of idiopathic transverse myelitis (ITM).

\section{Methods}

We compared whole-exome sequencing (WES) on germline samples from the 2 affected sisters with ITM with 3 of their healthy siblings.

\section{Results}

The 2 sisters with ITM both had acute onset of sensory loss in the legs, weakness, and bowel/ bladder dysfunction. The first developed ITM at age 15 years with a clinical nadir of complete paralysis, which slowly recovered over a few years. MRI demonstrated a persistent T2 lesion in the lower thoracic cord. The second developed ITM at age 50 years with a nadir of sensory loss from T6 down and paraparesis in the legs, associated with an MRI lesion at T6. She also made a partial recovery with treatment. Both sisters are homozygous for a missense variant in VPS37A (c.700C >A, p.Leu234Ile) identified by WES. We performed targeted sequencing of VPS37A in an additional 86 samples from patients with ITM and 175 with other diseases to investigate the p.Leu234Ile variant. We identified another patient with ITM homozygous for the same rare variant. No patients with multiple sclerosis, neuromyelitis optica, other neurologic conditions, or any healthy controls in public databases were homozygous for this variant.

\section{Conclusions}

A rare missense variant in VPS37A may predispose to development of ITM. Further studies are necessary to determine the frequency of this variant in the patient population and the mechanism through which it contributes to the risk of disease.
Correspondence

Dr. Levy:

mlevy@jhmi.edu 


\section{Glossary}

CIDR = classless interdomain routing; ESCRT $=$ endosomal sorting complexes required for transport; GATK = Genome Analysis Toolkit; HSP = hereditary spastic paraplegia; ITM = idiopathic transverse myelitis; SNV = single nucleotide variant; WES $=$ whole-exome sequencing.

Idiopathic transverse myelitis (ITM) is a monophasic autoimmune attack on the spinal cord, which leads to weakness, numbness, and bowel/bladder dysfunction; however, there is a spectrum of severity, and not all patients have this complete spectrum of deficits. ${ }^{1}$ The incidence is approximately 1 per million per year $(0.1 / 100,000)$, with a prevalence of approximately 7,500 Americans living with disability from their ITM today. ${ }^{2}$ There is a bimodal age distribution with peak onsets in the teenage years and in adulthood. Men and women are equally affected. The etiology of ITM is presumed to be an immune-mediated attack $2-3$ weeks after a systemic infection, possibly due to molecular mimicry. ${ }^{3}$

ITM is conventionally viewed as a sporadic disease, with no strong familial risk factors and no recognized genetic contribution to risk. Recently, we encountered a family of Polish origin with 2 sisters affected by ITM, 1 presenting originally in 1967 at age 15 years and 1 presenting in 2010 at age 50 years. This unusual occurrence prompted us to seek a genetic basis for ITM.

\section{Methods}

\section{Samples}

We extracted DNA samples from blood, saliva, or spinal fluid pellets using a DNA isolation kit (Qiagen DNeasy Blood \&Tissue Kit, cat \#69504).

\section{Whole-exome sequencing and analysis}

We performed whole-exome sequencing (WES) in 2 affected sisters and their 2 healthy brothers and 1 healthy sister. We captured the consensus coding sequence exonic regions and flanking intronic regions totaling $\sim 51 \mathrm{Mb}$ using the Agilent SureSelect XT kit and performed paired-end $100 \mathrm{bp}$ reads with the Illumina HiSeq 2500 platform. We aligned each read to the 1000 Genomes phase 2 (GRCh37) human genome reference with the Burrows-Wheeler Alignment v.0.5.10-tpx. ${ }^{4}$ Local realignment around indels and base call quality score recalibration were performed using the Genome Analysis Toolkit (GATK) v.2.3-9-ge5ebf34. Variant filtering was performed by the Variant Quality Score Recalibration method. ${ }^{6}$ For single nucleotide variants (SNVs), the annotations of MQRankSum, HaplotypeScore, QualByDepth, FisherStrand, RMSMappingQuality, and ReadPosRankSum were used in the adaptive error model $(6$ maximum Gaussians allowed, worst 3\% used for training the negative model). HapMap3.3 and Omni2.5 were used as training sites, with HapMap3.3 used as the truth set. SNVs were filtered to obtain all variants up to the 99th percentile of truth sites ( $1 \%$ false-negative rate). For indels, the annotations of QD, FS, HaplotypeScore, and ReadPosRankSum were used in the adaptive error model (4 maximum Gaussians allowed, worst $12 \%$ used for training the negative model; indels that had annotations more than $10 \mathrm{SD}$ from the mean were excluded from the Gaussian mixture model). A set of curated indels obtained from the GATK resource bundle (Mills_and_1000 G_gold_standard.indels.b37.vcf) were used as training and truth sites. Indels were filtered to obtain all variants up to the 95th percentile of truth sites ( $5 \%$ false-negative rate).

Using the PhenoDB Variant Analysis Tool of PhenoDB, ${ }^{8}$ we prioritized heterozygous, homozygous, and compound heterozygous rare functional variants (missense, nonsense, splice-site variants, and indels) shared by the proband and her affected sister but not present in the 2 healthy brothers and healthy sister. We excluded variants with a minor allele frequency of $>0.01$ in Single Nucleotide Polymorphism database 126, 129, and 131, the Exome Variant Server (release ESP6500SI-V2), 1000 Genomes Project, or among the samples sequenced at classless interdomain routing (CIDR) as part of the Baylor-Hopkins Center for Mendelian Genomics. We also excluded all variants found in our in-house controls (CIDRVar $51 \mathrm{Mb}$ ). We generated a list of heterozygous variants shared by the proband and her affected sister but not present in the 3 healthy brothers and sister; a list of homozygous variants shared by the proband and her affected sister but excluding variants that were homozygous in one of the healthy siblings and a list of compound heterozygous variants identified shared by the proband and her affected sister but excluding the genes that had the same set of variants in one of the unaffected siblings.

\section{PCR and Sanger sequencing of the VPS37A exon 6 variant}

We performed confirmatory Sanger sequencing on a 350-bp amplicon containing exon 6 of VPS37A (forward: aaggcagtgtgagatgtgaaga; reverse: tcccactaaggcaacaacaa primers) purified with the Agencourt AMPure XP automated PCR purification system and sequenced on an Applied Biosystems 3730xl DNA Analyzer.

\section{Standard protocol, approvals, registrations, and patient consents}

This project was approved by the Johns Hopkins University Institutional Review Board. All subjects consented to the publication of their cases.

\section{Results}

\section{Cases}

Family 1 , sister 1 is currently 64 years old but woke up at age 15 years with abrupt lower extremity weakness and sensory 
loss. She recalls no immediate antecedent illness. She was treated with corticosteroids, and she made a slow and steady recovery over 3 years from a nadir of being bedbound to being able to ambulate without assistance. MRI performed at age 53 years still showed a T2 hyperintense lesion at T8/T9 with no brain lesions (figure 1A). No additional neurologic events have occurred in the 49 years since the acute onset of her ITM. Family 1, sister 2 is currently 59 years old and was 51 when she developed acute onset of low back pain, followed by sensory loss in both legs, mild leg weakness, and urinary incontinence. She recalls no immediate antecedent illness. Examination revealed normal strength and a sensory level at T10. Her spinal cord MRI revealed a nonenhancing T2 hyperintense lesion at T4/T5 (figure 1B). She was treated with a 1-month course of oral corticosteroids and over the next 6 months made a near full recovery except for a persistent mild leg weakness and sensory level on examination. Spinal fluid analysis 4 months after the acute onset revealed no pleocytosis nor oligoclonal bands, and serology was negative for autoantibodies. Brain MRI was unremarkable. No further

Figure 1 Spine MRI, sagittal and axial views of patients with idiopathic transverse myelitis with VPS37A mutations
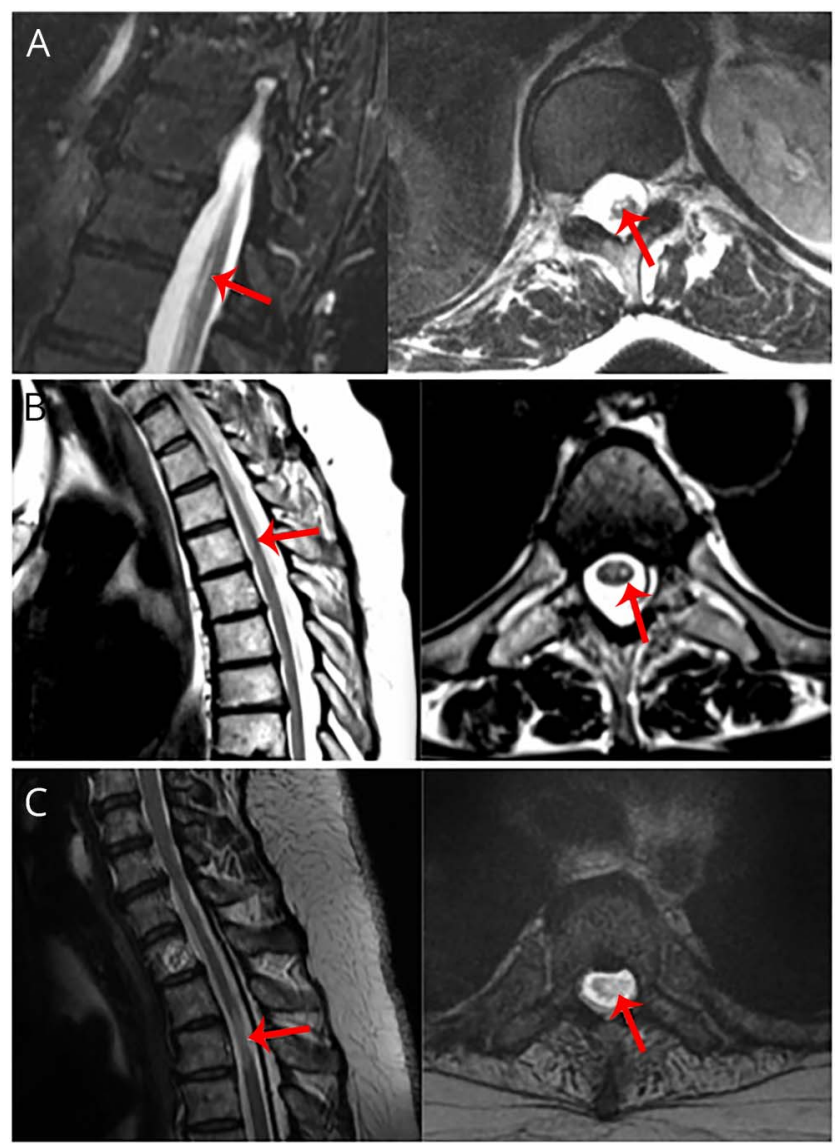

(A) Sister 1 with idiopathic transverse myelitis (ITM) shows a T2 hyperintense lesion 38 years after the onset at T8/T9 (arrows). (B) Sister 2 with ITM shows an acute T2 hyperintense lesion at T4/T5 (arrows). (C) Unrelated patient with ITM shows an acute T2 hyperintense lesion at T3 (arrows). neurologic events have occurred in the 8 years since her ITM. The sisters have 2 brothers and another sister, all healthy. The family history revealed no consanguinity; the parents were both of Polish origin. There was no other history of ITM.

\section{DNA sequence results}

WES and analysis on germline DNA from the 2 affected sisters and their 2 healthy brothers and 1 healthy sister revealed a missense, homozygous variant in VPS37A, isoform 1 (c.700C>A, p.Leu234Ile, rs150912414, ch8:17276454). The leucine at this position is remarkably well conserved among all known eukaryotes (figure 2). One of the healthy brothers was heterozygous for this variant. There were no additional homozygous or compound heterozygous candidate variants identified that segregated appropriately.

\section{Screening other patients with ITM}

In an additional 86 individuals presenting with ITM screened for this variant in VPS37A, we identified a third patient

Figure 2 DNA sequence analysis of VPS37A
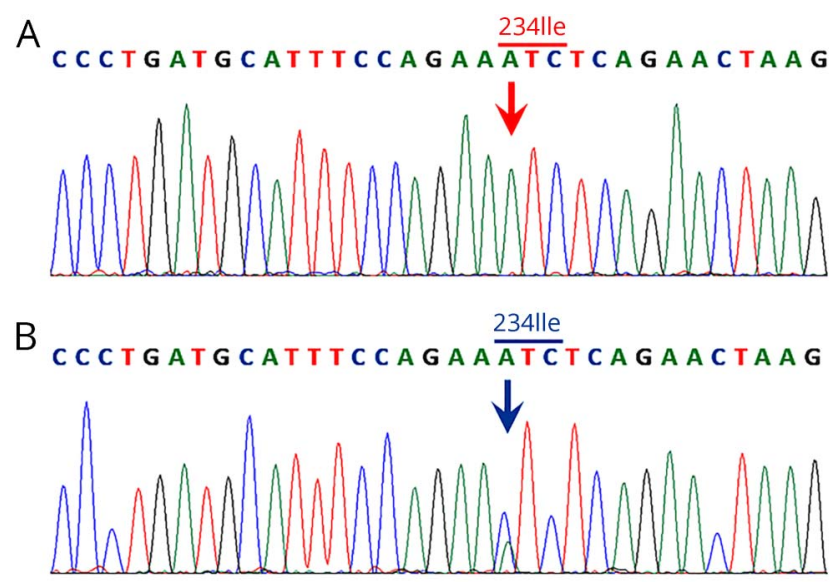

C C C C T G A T G C A T T T C C A G A A $\frac{\operatorname{LeU} 234}{\mathbf{C} \text { T C }}$ T C A G A A C TA A G

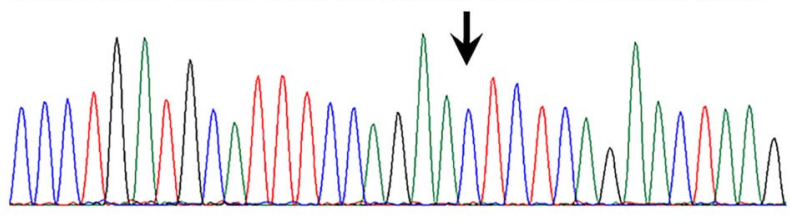

D
Homo sapiens Callithrix jacchus Rattus norvegicus Mus musculus Gallus gallus

Xenopus laevis

Danio rerio
Pro Asp Ala Phe Pro Glu Leu Ser Glu Leu Pro Asp Ala Phe Pro Glu Leu Ser Glu Leu Pro Asp Ala Phe Pro Glu Leu Ser Glu Leu Pro Asp Ala Phe Pro Glu Leu Ser Glu Leu Pro Asp Thr Phe Pro Glu Leu Leu Glu Leu Pro Glu Thr Phe Ser Glu Leu Leu Glu Leu Pro Asp Thr Phe Ser Glu Leu Ser Glu Met
(A) Electropherogram of the patient reveals a nonsynonymous homozygous C-to-A substitution (red arrow) at position 700 bp of VPS37A (c.700C $>\mathrm{A}$, p.Leu234lle). (B) Electropherogram reveals a heterozygous c.700C $>A$ mutation (blue arrow) in patients without idiopathic transverse myelitis. (C) Representative electropherogram of VPS37A from normal controls (black arrow). (D) p.Leu234 residue is highly conserved and found in evolutionary distant orthologs down to zebrafish. The sequences were derived from GenBank records with the following accession numbers: Homo sapiens (NP 689628.2), Callithrix jacchus (marmoset; XP 008977480.1), Rattus norvegicus (Rat; NP_001020038.1), Mus musculus (house mouse; NP_291038.2), Gallus gallus (chicken; XP_420687.1), Xenopus laevis (African clawed frog; XP_018105271.1), and Danio rerio (zebrafish; NP_956284.1). 
homozygous for the same VPS37A variant. This woman is currently 55 years old who developed acute mid-thoracic back pain and bilateral numbness in her toes when she was 50 years old. An examination revealed mild left leg weakness. Her spine MRI revealed a T2 hyperintense lesion at T3. Her brain MRI was normal (figure 1C). Spinal fluid analysis was not performed, and serology was negative for autoantibodies. She was treated with steroids and made a partial recovery with ongoing back pain and left leg spasms. No further neurologic events have occurred in the ensuing 5 years. She is of Irish/Scottish origin, denies consanguinity, and has no family history of ITM. Her symptomatic sister is heterozygous for the variant.

\section{Screening among patients with nontransverse myelitis}

We screened an additional 175 individuals with the following diagnoses: multiple sclerosis $(\mathrm{n}=25)$, neuromyelitis optica $(n=25)$, healthy controls $(n=25)$, and other neurologic disease $(n=100)$. None were homozygous for the VPS37A-L234I variant; however, we identified an L234I heterozygote who was a 70-year-old man with an inflammatory bony lesion of his skull base with no history of myelopathy (figure 3).

\section{Analysis}

VPS37A-L234I is found in heterozygosity in 4/10,000 individuals in the National Heart, Lung, and Blood Institute Exome Sequencing Project, in 49/120,880 individuals in the genome Aggregation Database, and 30/10,000 European descent individuals who participated in the ClinSeq project. $^{9-11}$ Given the allele frequency across multiple control data sets, the expected frequency of homozygosity for this variant is extremely small, $\sim 1.6 \times 10 \mathrm{e}^{-9}$. After forming the hypothesis that this variant is pathogenic based on family 1 with the 2 affected sisters, we tested it in our small cohort $(\mathrm{n}=$ 86) of similarly affected unrelated patients and found an additional VPS37A-L234I homozygote. Considering the genome Aggregation Database with no reported homozygotes in 120,880 individuals, our result identifying 1 homozygote in only 238 patients with neuro-immune phenotypes rejects the null hypothesis (Fisher exact test, $p<0.002$ ) and supports that homozygosity for VPS37A-L234I confers susceptibility for the 3 individuals with ITM. Significance is higher if calculated only for the 88 patients with ITM $(p<0.00072)$.

\section{Discussion}

We identified a rare missense variant, L234I, in VPS37A in 3 patients from 2 families with ITM. The rarity of this variant among the general population and the occurrence in homozygosity in affected members of 2 unrelated families implicate this variant in susceptibility to ITM. Based on the screening we conducted among a total of 88 unrelated patients with ITM, 25 with multiple sclerosis, 25 with neuromyelitis optica, and 100 with other neurologic diseases, we estimate that up to $3 \%$ of ITM may be associated with this genetic variant. We are in the process of validating the usefulness of VPS37A
Figure 3 Pedigree of familial idiopathic transverse myelitis

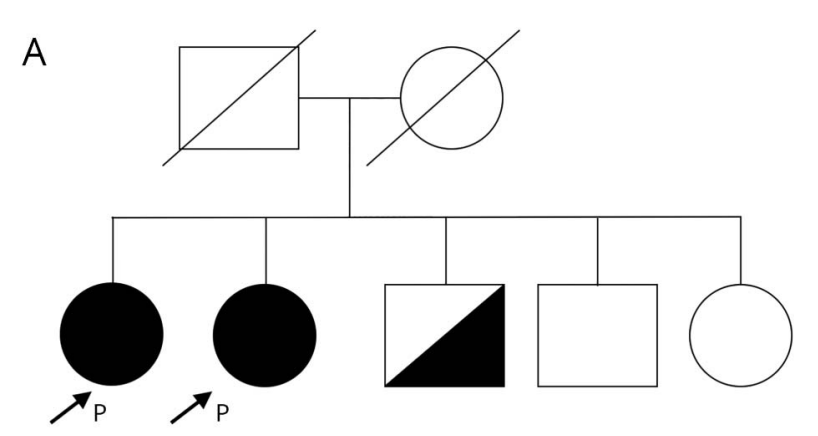

B

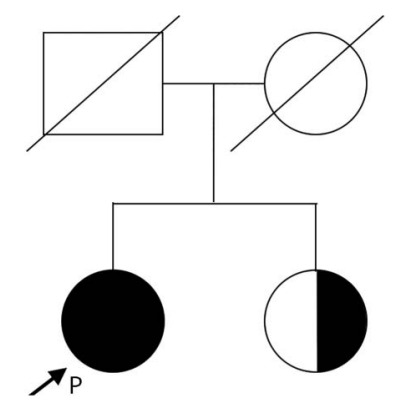

(A) Family with 2 affected sisters who are both positive for the VPS37A mutation (closed circles, $P$ arrows) and 2 healthy brothers, one of whom is a carrier (half-filled square) and one of whom is not (clear square) and a healthy sister (clear circle). Their parents are not available to be tested. (B) A patient with idiopathic transverse myelitis was screened and found to have the mutation, with no known family history (closed circle, P arrow). Her sister is a healthy carrier (half-filled circle).

sequencing in patients with ITM, especially those with a positive family history.

VPS37A encodes a component of endosomal sorting complexes required for transport (ESCRT)-1, one of a set of heteromeric complexes (ESCRT-0 to ESCRT-3) that are involved in membrane remodeling processes including endosomal sorting, multivesicular body biogenesis, nuclear pore homeostasis, and budding of virus-containing vesicles into the extracellular space. ${ }^{12-14}$ The 4 ESCRT complexes comprise more than 30 proteins, and defects in several of these have been associated with neurodegenerative diseases including Alzheimer disease, motor neuron disease, and hereditary spastic paraplegia (HSP). ${ }^{15}$ Relevant to our results, a previous study reported 9 patients in 2 consanguineous Middle Eastern families with autosomal recessive HSP (spastic paraparesis 53, OMIM 614898). ${ }^{16}$ Affected individuals were homozygous for a VPS37A missense variant Lys382Asp in exon 11. The clinical phenotype included the onset of spastic paraparesis in their first 3 years of life, accompanied by intellectual disability, kyphosis, and pectus carinatum. ${ }^{16}$ There was a progressive, nonimmune-mediated, degenerative course, with normal MRI findings of the spine in at least 2. By contrast, the clinical phenotype of our patients featured a single, immune-mediated attack of the spinal cord 
Table Autoimmune conditions associated with a monogenetic mutation

\begin{tabular}{ll}
\hline Autoimmune condition & Genetic mutation(s) \\
\hline Autoimmune polyglandular syndrome type 1 & AIRE \\
\hline Immune dysregulation, polyendocrinopathy, enteropathy, and X-linked syndrome & FOXP3 \\
\hline Aicardi-Goutières syndrome & TREX1, RNASE H2, SAMHD1, and ADAR1 \\
\hline C1q deficiency & C1QA, C1QB, or C1QC \\
\hline Spondyloenchondrodysplasia with immune dysregulation & ACP5 \\
\hline Type 1 diabetes and autoimmune thyroiditis & AIRE, FOXP3, IL2RA, ITCH, LRBA, STAT1, and STAT3 \\
\hline CNS infection risk & Genetic mutation(s) \\
\hline Herpes simplex encephalitis & TLR3, UNC93B1, TRIF, TRAF3, and TBK1 \\
\hline West Nile virus & CCR5 \\
\hline Infection-triggered familial acute necrotizing encephalopathy & RANBP2 \\
\hline
\end{tabular}

Abbreviations: AIRE = autoimmune regulator; FOXP3 = forkhead box protein 3.

confirmed by a focal MRI lesion in the spinal cord, followed by a stable or slowly improving clinical course after treatment with corticosteroids.

The mechanism by which a variant in VPS37A results in an immune-mediated attack confined to the spinal cord is unknown; however, there are a growing list of autoimmune conditions associated with monogenetic variants (table). There are also monogenic disorders that impart a risk to infections such as herpes simplex virus encephalitis. These variants do not cause encephalitis, but rather they impair the normal innate immune response to the herpes simplex virus. Thus, the variant confers susceptibility to the environmental exposure to herpes simplex virus, and the phenotype is generated by a genetic defect that is exposed or triggered by the environmental variable. Of interest, a preceding systemic infection has been documented in approximately two-thirds of ITM cases, ${ }^{2}$ suggesting that an environmental insult may be necessary to expose the genetic defect by generating an abnormal immunopathogenic response. Similar "conditional" mendelian phenotypes include the well-known hemolytic anemia (OMIM 300908) resulting from mutations in the G6PD gene in patients exposed to fava beans and other infections in the nervous system genetically susceptible individuals (table).

Our study is limited to this single variant in exon 6 of VPS37A. Although an amino acid substitution of leucine to isoleucine at this position is predicted to be deleterious, we have not analyzed VPS37A protein expression in these patients. In addition, the healthy brothers and sisters may develop ITM in the future. We are also in the process of screening patients with ITM for variants in other regions of VPS37A and other components of the ESCRT system. Mouse models homozygous for the VPS37A-Leu234Ile variant may shed light on the mechanism of how a genetic variant confers vulnerability to a monophasic immune-mediated process in the spinal cord.

\section{Author contributions}

Maureen A. Mealy, Tai-Seung Nam, Santiago J. Pardo, Carlos A. Pardo, David Valle, Kathleen H. Burns, and Michael Levy: contributed to the design and conceptualization of the study, analysis and interpretation of the data, and drafting of the manuscript. Tai-Seung Nam and Santiago J. Pardo,: performed the experiments.

\section{Study funding}

This study was funded by the National Institutes of Health, K08 NS078555 (M.L.) and by the Baylor-Hopkins Center for Mendelian Genetics, NHGRI-1U54HG006542 (D.V.). The funding source had no role in study design; in the collection, analysis, and interpretation of data; in the writing of the report; or in the decision to submit the article for publication.

\section{Disclosure}

M.A. Mealy has received speaker honoraria from the Consortium of Multiple Sclerosis Centers and has received research support from the NIH. T.-S. Nam and S.J. Pardo report no disclosures. C.A. Pardo has served on the scientific advisory board of the Transverse Myelitis Association and has received research support from Acorda Pharmaceuticals, Chugai Pharmaceutical Co. LTD, MedImmune, the NIH, and the Bart McLean Fund for Neuroimmunology Research (Project Restore Transverse Myelitis Association). N.L. Sobreira receives research support from the NIH, the Guthy Jackson Charitable Foundation, Viropharma, Acorda, Sanofi, NeuralStem, and Genentech and serves as a consultant 
for Alexion, Chugai Pharmaceuticals, GlaxoSmithKline, and MedImmune. D. Avramopoulos serves on the editorial boards of Frontiers in Genetics and Molecular Neuropsychiatry; receives publishing royalties from Elsevier Academic Press; receives research support from the NIH, the Guthy Jackson Charitable Foundation, Viropharma, Acorda, Sanofi, NeuralStem, and Genentech; and serves as a consultant for Alexion, Chugai Pharmaceuticals, GlaxoSmithKline, and MedImmune. D. Valle receives publishing royalties from McGraw-Hill; has been a consultant for Third Rock Ventures; receives research support from the NIH, the Guthy Jackson Charitable Foundation, Viropharma, Acorda, Sanofi, NeuralStem, and Genentech; and serves as a consultant for Alexion, Chugai Pharmaceuticals, GlaxoSmithKline, and MedImmune. K.H. Burns receives research support from JUNO Therapeutics, the NIH, and the NMSS. M. Levy has served on the scientific advisory boards of Asterias, Chugai, and Alexion; has served on the editorial board of Multiple Sclerosis and Related Disorders; holds patents for Aquaporin-4 sequence that elicits pathogenic $\mathrm{T}$ cell response in animal model of neuromyelitis optica and for Use of peptide for diagnostic and therapeutic developments; has been a consultant for Guidepoint Global, Gerson Lehrman Group, and Cowen Group; and receives research support from Viropharma/Shire, Acorda, ApoPharma, Sanofi Genzyme, Alnylam, Alexion, Terumo BCT, the Guthy Jackson Charitable Foundation, Viropharma, Acorda, NeuralStem, and Genentech; and serves as a consultant for Alexion, Chugai Pharmaceuticals, GlaxoSmithKline, and MedImmune. Full disclosure form information provided by the authors is available with the full text of this article at Neurology.org/NG.
Received July 21, 2017. Accepted in final form November 20, 2017.

\section{References}

1. Krishnan C, Kaplin AI, Deshpande DM, Pardo CA, Kerr DA. Transverse myelitis: pathogenesis, diagnosis and treatment. Front Biosci 2004;9:1483-1499.

2. Frohman EM, Wingerchuk DM. Clinical practice: transverse myelitis. N Engl J Med 2010;363:564-572.

3. Kaplin AI, Krishnan C, Deshpande DM, Pardo CA, Kerr DA. Diagnosis and management of acute myelopathies. Neurologist 2005;11:2-18.

4. Li H, Durbin R. Fast and accurate short read alignment with Burrows-Wheeler transform. Bioinformatics 2009;25:1754-1760.

5. McKenna A, Hanna M, Banks E, et al. The genome analysis toolkit: a MapReduce framework for analyzing next-generation DNA sequencing data. Genome Res 2010; 20:1297-1303.

6. DePristo MA, Banks E, Poplin R, et al. A framework for variation discovery and genotyping using next-generation DNA sequencing data. Nat Genet 2011;43: 491-498.

7. You J, Sobreira NL, Gable DL, et al. A syndromic intellectual disability disorder caused by variants in TELO2, a gene encoding a component of the TTT complex. Am J Hum Genet 2016;98:909-918.

8. Sobreira N, Schiettecatte F, Boehm C, Valle D, Hamosh A. New tools for Mendelian disease gene identification: PhenoDB variant analysis module; and GeneMatcher, a web-based tool for linking investigators with an interest in the same gene. Hum Mutat 2015;36:425-431.

9. Biesecker LG, Mullikin JC, Facio FM, et al. The ClinSeq Project: piloting large-scale genome sequencing for research in genomic medicine. Genome Res 2009;19: 1665-1674.

10. Lek M, Karczewski KJ, Minikel EV, et al. Analysis of protein-coding genetic variation in 60,706 humans. Nature 2016;536:285-291.

11. Server EV. NHLBI Exome Sequencing Project (ESP) [online]. Available at: http:// evs.gs.washington.edu/EVS/. Accessed May 2017.

12. Campsteijn C, Vietri M, Stenmark H. Novel ESCRT functions in cell biology: spiraling out of control? Curr Opin Cell Biol 2016;41:1-8.

13. Olmos Y, Carlton JG. The ESCRT machinery: new roles at new holes. Curr Opin Cell Biol 2016;38:1-11.

14. Wunderley L, Brownhill K, Stefani F, Tabernero L, Woodman P. The molecular basis for selective assembly of the UBAP1-containing endosome-specific ESCRT-I complex. J Cell Sci 2014;127:663-672.

15. Fink JK. Hereditary spastic paraplegia: clinico-pathologic features and emerging molecular mechanisms. Acta Neuropathol 2013;126:307-328.

16. Zivony-Elboum Y, Westbroek W, Kfir N, et al. A founder mutation in Vps37A causes autosomal recessive complex hereditary spastic paraparesis. J Med Genet 2012;49: $462-472$. 


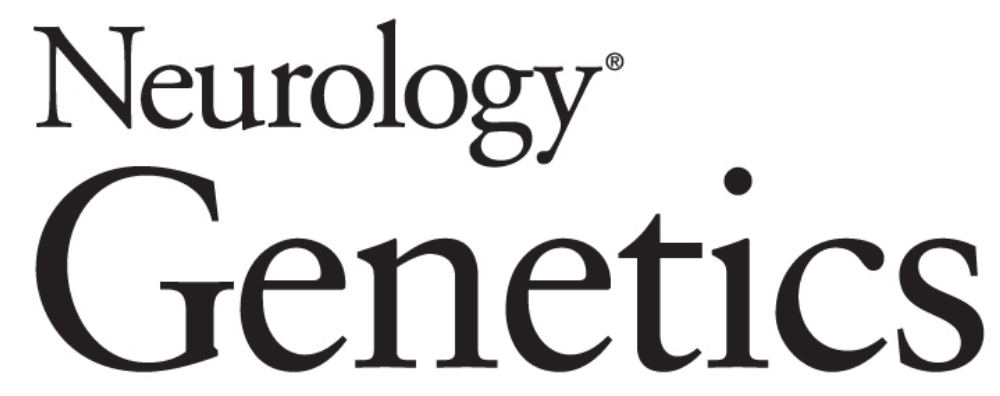

Familial monophasic acute transverse myelitis due to the pathogenic variant in VPS37A Maureen A. Mealy, Tai-Seung Nam, Santiago J. Pardo, et al. Neurol Genet 2018;4; DOI 10.1212/NXG.0000000000000213

This information is current as of January 30, 2018

\section{Updated Information \& Services}

References

Citations

Subspecialty Collections

Permissions \& Licensing

Reprints including high resolution figures, can be found at: http://ng.neurology.org/content/4/1/e213.full.html

This article cites 15 articles, 4 of which you can access for free at: http://ng.neurology.org/content/4/1/e213.full.html\#\#ref-list-1

This article has been cited by 1 HighWire-hosted articles: http://ng.neurology.org/content/4/1/e213.full.html\#\#otherarticles

This article, along with others on similar topics, appears in the following collection(s):

Genetic linkage

http://ng.neurology.org//cgi/collection/genetic_linkage

Transverse myelitis

http://ng.neurology.org//cgi/collection/transverse_myelitis

Information about reproducing this article in parts (figures,tables) or in its entirety can be found online at:

http://ng.neurology.org/misc/about.xhtml\#permissions

Information about ordering reprints can be found online: http://ng.neurology.org/misc/addir.xhtml\#reprintsus

Neurol Genet is an official journal of the American Academy of Neurology. Published since April 2015, it is an open-access, online-only, continuous publication journal. Copyright Copyright @ 2018 The Author(s). Published by Wolters Kluwer Health, Inc. on behalf of the American Academy of Neurology.. All rights reserved. Online ISSN: 2376-7839.

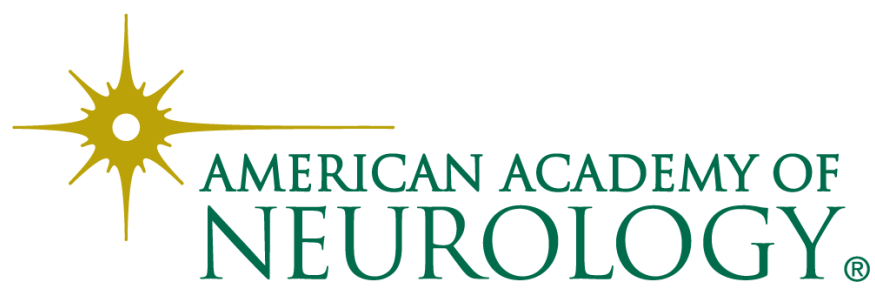

